\title{
Research Article \\ Open Access \\ Reporting 5 Cases of Forgotten Ureteral Stent Treated by Endourological Approaches and Citrate Alkaline Therapy
}

\author{
Dr. Yasin ldweini, Ph.D., MD, FEBU, \\ Senior Consultant and Chairman of Urology \\ Urology department Al- Bashir Teaching Hospital Amman Jordan \\ yasin_idweini@hotmail.com
}

\begin{abstract}
Purpose: This research it intended to present our experience in various endourological approaches and citrate alkaline therapy for treating forgotten encrusted ureteral stents.

Material and Methods: During the term starting from January 2010 till December 2013, five patients; 2 males and 3 females; with encrusted urethral stents, were studied. The average indwelling time of the stent was 5 years (range is 2 to 13 years). Mean ages were 37 years old. Kidney function test, urine culture, coronal computerized tomography (C/T) scan without contrast, plain- film radiography, intravenous urogram and renal isotopes scan were used for renal function assessment.

Results: The entire stent of all patients sample was encrusted. An Extracorporeal shock waves lithotripsy (ESWL) was performed for all, in addition to citrate alkaline medication in order to trying stopping the progress of encrustation and the growth of stones. Cystolithotripsy was used to manage the distal coil of the encrusted stents and in some cases we used cystolitholapaxy to crush bladder stones in addition to heavily encrusted stents. All patients rendered stone and stent free. One patient remained in the follow up symptomatic with loin pain, and thus he needed open surgery of nephrectomy due to impaired non-function kidney, with the histopathology report of malakoplakia of the kidney; which is rare inflammatory disease that is associated with Escherichia Coli infection.

Conclusion: Endourological management and alkaline medication of forgotten stents is highly successful and often there is no need for open surgery.
\end{abstract}

Keywords: Forgotten stents; urethral; lithotripsy; lithiasis; endourology; Citrate.

\section{INTRODUCTION}

In 1967 Zimskind was the first to describe endoscopic stent placement to relieve urethral obstruction (1). Stents are hollow tubes with a coil at each end, which is inserted through the bladder; usually into the ureter and thence into the renal pelvis

Urethral stents are widely used in urological practice. They are designed to bypass a ureteric obstruction due to intrinsic or extrinsic causes (e.g. stone, malignancies, strictures etc.)or draining the kidney (e.g. post renal surgery) (1-5).They are also placed after iatrogenic injuries of the ureter and before any complex abdomen procedure to identifying and protecting ureters (6).Complications of wide spread usage of stents are stent encrustation, stent fragmentation, stone formation and recurrent urinary tract infection $(7,8)$. There are many methods of treatment combinations of extracorporeal shock wave lithotripsy, cystolithotripsy, retrograde 
Reporting 5 Cases of Forgotten Ureteral Stent Treated by Endourological Approaches and Citrate Alkaline Therapy

ureteroscopy with intra-corporeal lithotripsy, percutaneous nephrolithotomy and open surgery has been used as to removal of these encrusted stents (9-14).

\section{Forgotten Stent}

rare, but potentially is very serious as the biofilm may become encrusted with stone, and further making, technically,the removal process very difficult.

We are reporting herein these cases to highlighting the possible serious complications, with long-term indwelling stents, in patients who do not attend to follow up the forgotten stent that frequently originates complications.

These delayed complications including hydronephrosis, encrustation, blockage, stuck stents, stent migration, stent knotting, fracture, spontaneous fragmentation and stenturia; pose a management and legal dilemma.

Certain precautions and guidelines must be observed whenever JJ stent is used .The stent is a double -edged sword and thus routine usage thereof is not justified; at least where endourological facilities are not available in institutions.

We are reporting 5 cases of forgotten JJ ureteral stent which had been placed long time ago; four patients who received urolithiasis therapy and one patient underwent pyeloplasty surgery in $\mathrm{Al}$-BashirHospital.

Males to females' ratio is 2:3. Clinical picture was symptomatic in 3 patients with flank pain and recurrent UTI, while two patients were asymptomatic. Forgotten stent duration between 2-13 years, is ranged at 5-years. Mean age was 37 years. Radiography of the kidneys, ureters, bladder and computed tomography demonstrated stone encrustation over the whole JJ stent. Kidney function test, renal isotopes scan and urine cultures were performed before the treatment. Alkaline citrate therapy with potassium sodium hydrogen citrate granules, as stone formation inhibitors was taken from the first beginning of treatment, ESWL,cystolithotripsy and ureteroscopic lithotripsy were performed to remove JJ performed.

\section{First case}

A 38- year's old, married female patient suffering from high fever up to $39^{\circ} \mathrm{C}$ and severe right flank pain; was admitted to the emergency room at $\mathrm{Al}$-Bashir Hospital. The first impression of the case was right perinephretic abscess by ultrasound and CT scan. Right flank percutaneous drainage was done and yielded pus-like fluid.

Fever and flank pain subsided much after drainage, and after 3 days later, urine came out by drain, which means that spontaneous rupture of one calyx occurred and fistula formation between calyx and Para- renal space is performed.

JJ stent was inserted in right ureter and Foley's catheter was also inserted to prevent reflux of the urine to the kidney and KUB showed right ureteric stone beside JJ in situ. Urine stopped to come out by drainage after two days. Then drain removed, patient was discharged in good condition to be seen, one month later, by ESWL for her ureteric stone. After ESWL therapy,the patient did not follow -up her case anymore, even though she should follow up after one week from her discharge to receive the result of therapy by KUB X-Rays.

Many communication trials were made with the patient to enable us dealing with the JJ stent but to no avail. After two years we received telephone call from her father who brought her to our outpatient clinic, which imposed on us to start the medical treatment from the first beginning.

We have tested her kidney function which was within normal value, and further her urine culture was free from bacterial growth.

The renal isotopes scan DMSA showed Right kidney scarred \& Lt kidney normal. 
Reporting 5 Cases of Forgotten Ureteral Stent Treated by Endourological Approaches and Citrate Alkaline Therapy

\section{Split function}

Rt kidney function was 29\% Lt kidney function was 71\%. The CT scan showed encrusted stent and ureteric stone. Thus multiple modality of treatment was started. ESWL of the encrusted upper coil of the stent was performed. Alkaline therapy started from the first beginning, with citrate sodium and potassium medication to decrease encrustation and stone formation. Lithotripsy of the lower encrusted coil was performed. Ureteroscopy with lithotripsy of ureteric stone and removal of encrusted stent was also done. The duration of treatment was extended to six months. The patient was subject to many follow ups but she remained symptomatic with loin pain. Second renal isotopes' scan DMSA was performed after completing the treatment; that displayed impaired non function of the Rt kidney, and thus it required nephrectomy to be done. Further the histopathology reported malakoplakia of the kidney; which is a rare inflammatory disease associated with Escherichia Coli infection (Granulomatous interstitial nephritis caused by kidney parenchymal malakoplakia). Fig.(1-2).
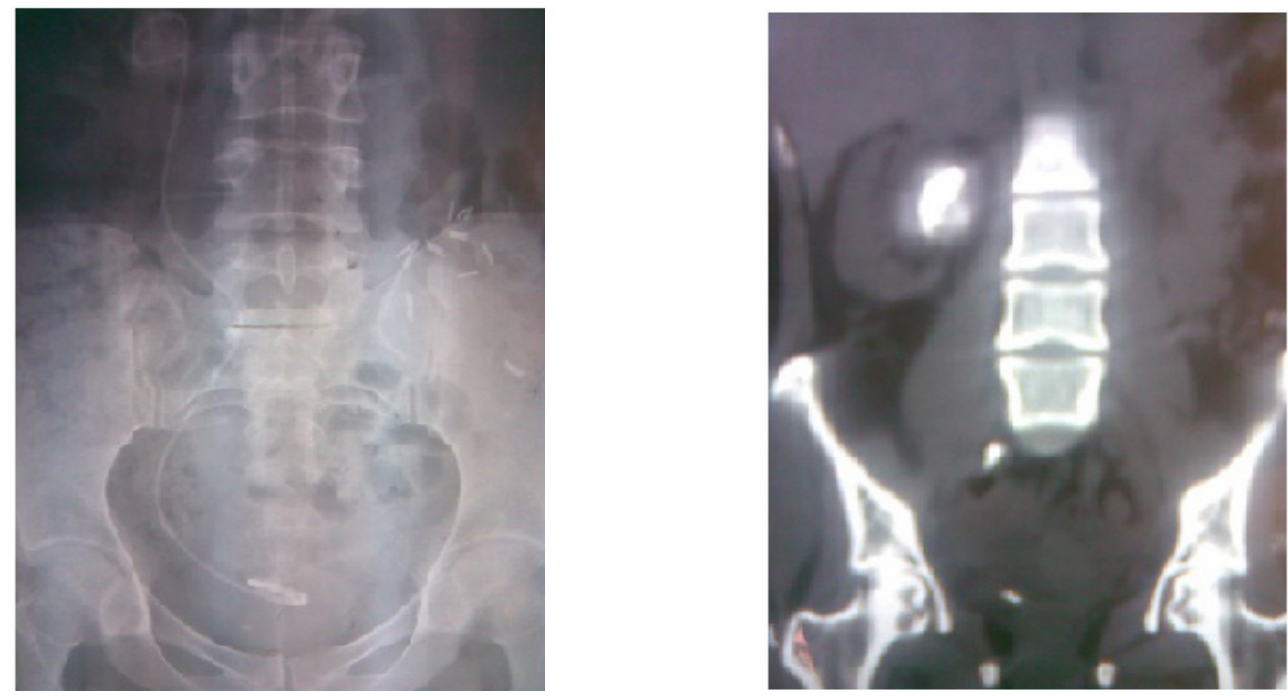

Fig1. X-Ray of the first Case showed heavy encrusted stents beside ureteric stone.
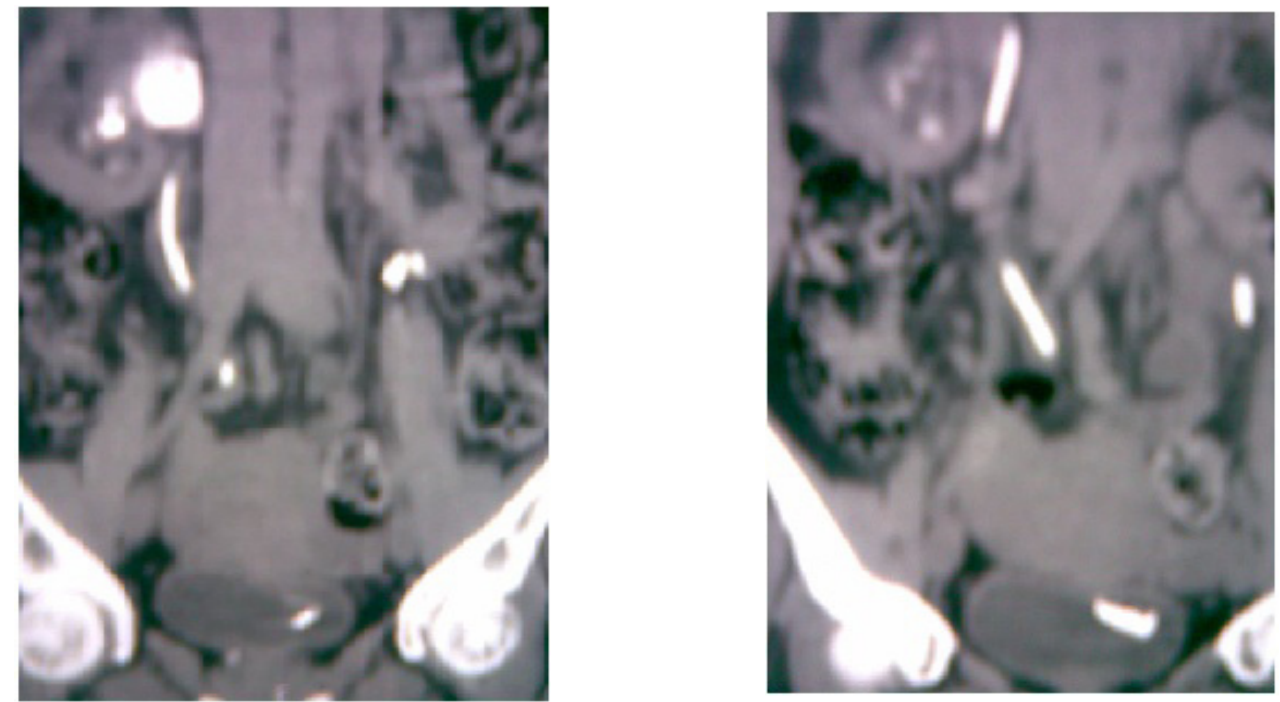

Fig2. CT scan of the first case; Heavy forgotten encrusted stent 
Reporting 5 Cases of Forgotten Ureteral Stent Treated by Endourological Approaches and Citrate Alkaline Therapy

\section{$2^{\mathrm{ND}}$ CASE}

A 33 years female patient underwent, on 9-October- 2009,RtPyeloplasty Andersen- Hynes due to pelvi-ureteric junction (PUJ) obstruction with JJ insertion. In November 2009 she was pregnant and Delivery was in August 2010.

She presented in June 2013 with forgotten stent (for 3.5-years duration),

4-episodes of ESWLs and Alkaline therapy (Uralyt U) were performed. The stent performed was removed after 4 months of treatment commencement. Fig. $(3,4,5)$.
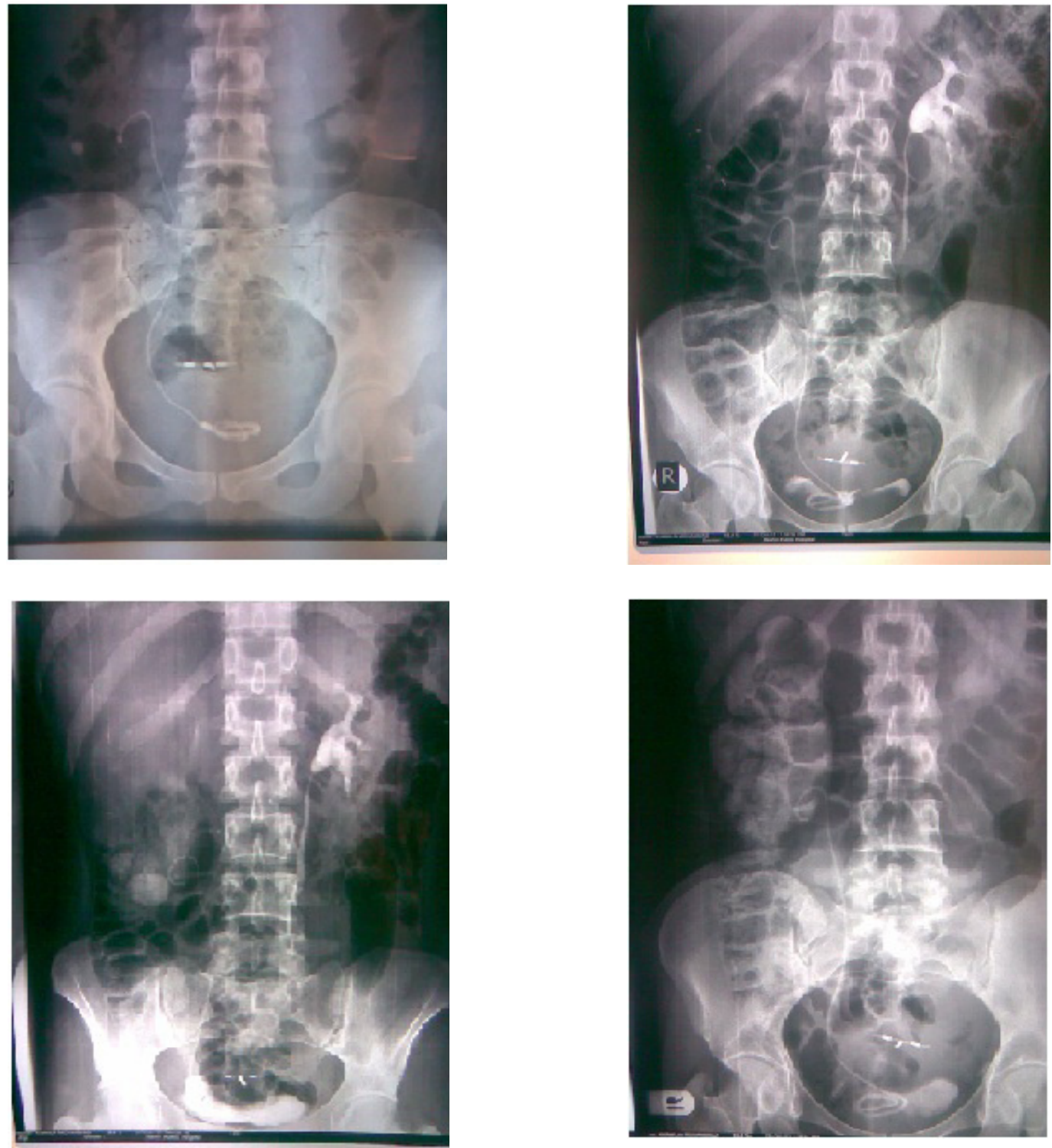

Fig3. IVU of the 2 nd case showed encrusted forgotten stent in pyeloplasty 
Reporting 5 Cases of Forgotten Ureteral Stent Treated by Endourological Approaches and Citrate Alkaline Therapy

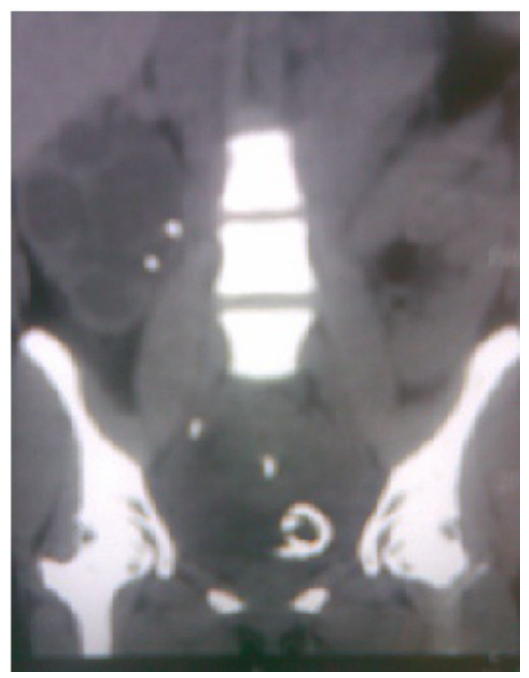

Fig4. CT scan: encrusted forgotten stent of the second case
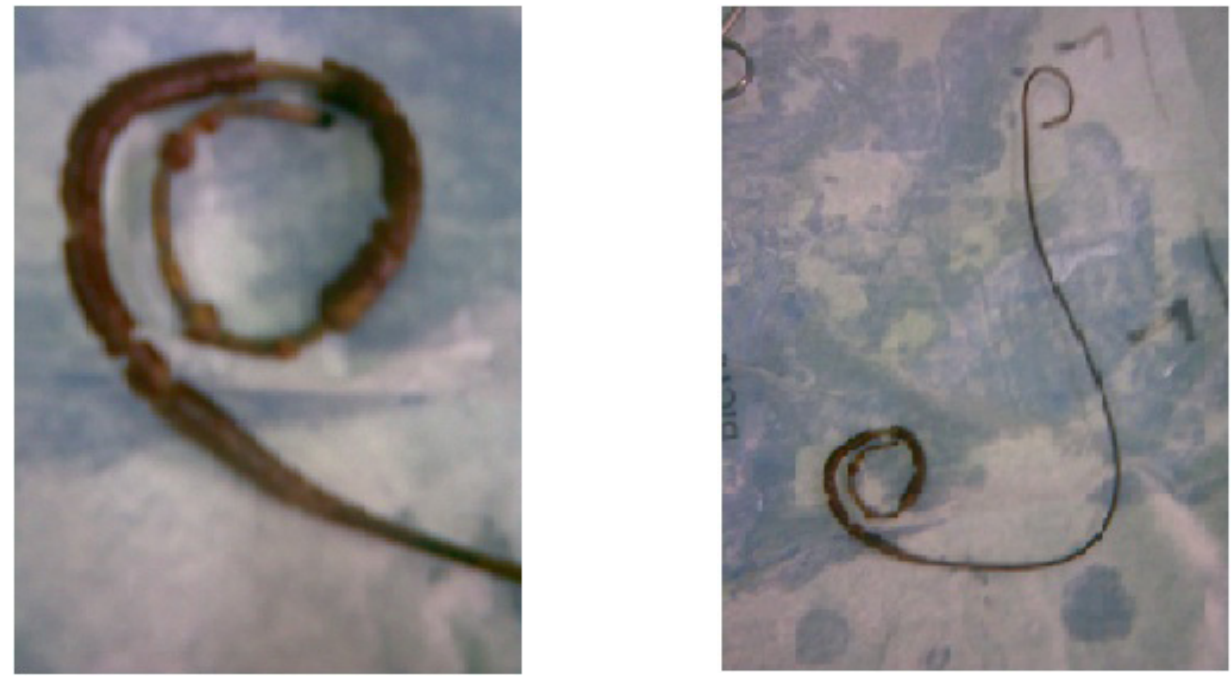

Fig5. forgotten encrusted stent after removal

\section{$3^{\mathrm{RD}}$ CASE}

A 38-years old female patient suffering from big stone at left kidney (lower moiety of pelvis in uncompleted duplication) JJ stent was inserted and two episodes of ESWLs were performed.

Said patient did not check-up again at our clinic for four years' time in order to remove the JJ stent, during which the stent with encrustation migrated upward to ureter. She admitted to our department suffering loin pain. She underwent alkaline therapy; many episodes of ESWLs as to the encrusted stent and ureteroscopy also were performed followed by removal of JJ stent. Treatment duration of the foregoing lasted for 4 months. (Fig.6) 
Reporting 5 Cases of Forgotten Ureteral Stent Treated by Endourological Approaches and Citrate Alkaline Therapy
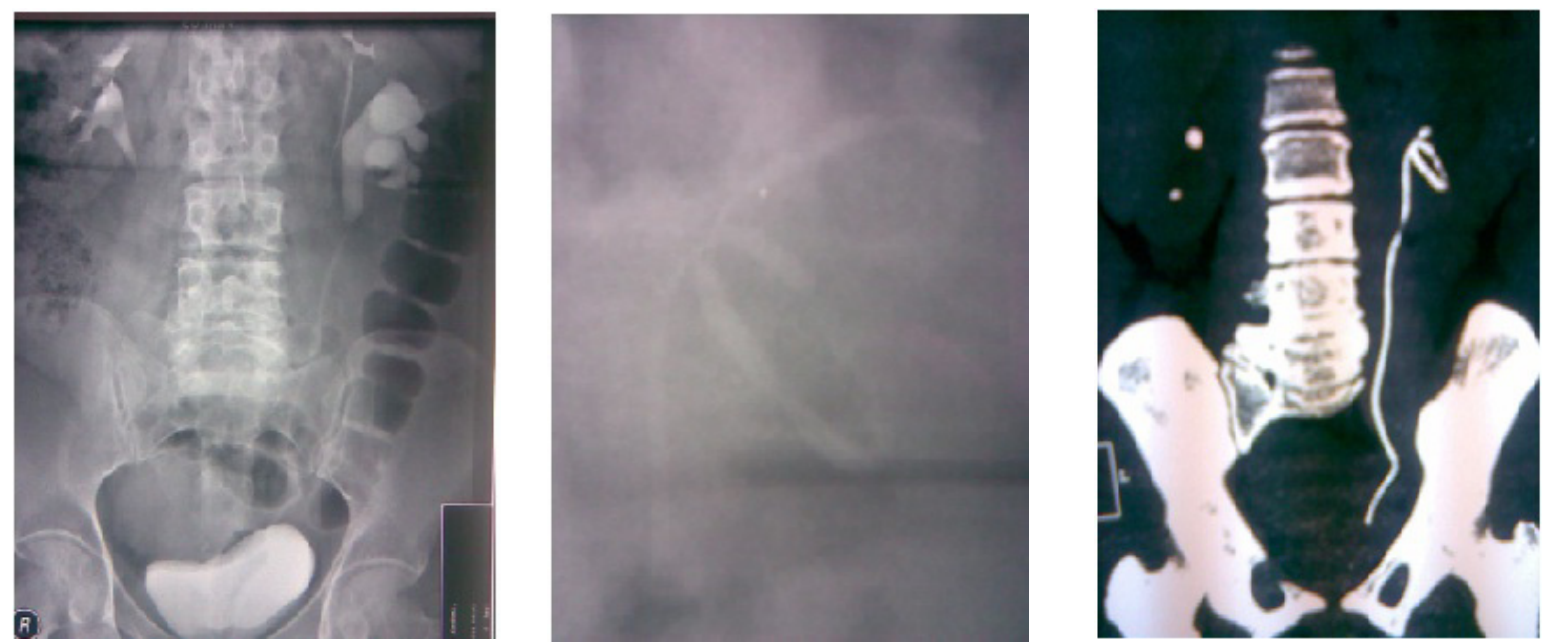

Fig6. IVU and CT scan of the third case: showed migrated encrusted stent upward.

The remained two cases included two men who suffer urolithiasis and forgotten stents. Stents were calcified, the mean duration since stent placement was for 7 years. ESWL, cystoscopic electrohydraulic lithotripsy, cystolitholopaxy, ureteroscopy with lithotripsy and citrate medication were performed. They needed all foregoing procedures to render them stones and stent-free. Treatment duration was 8 months. (Fig.7).
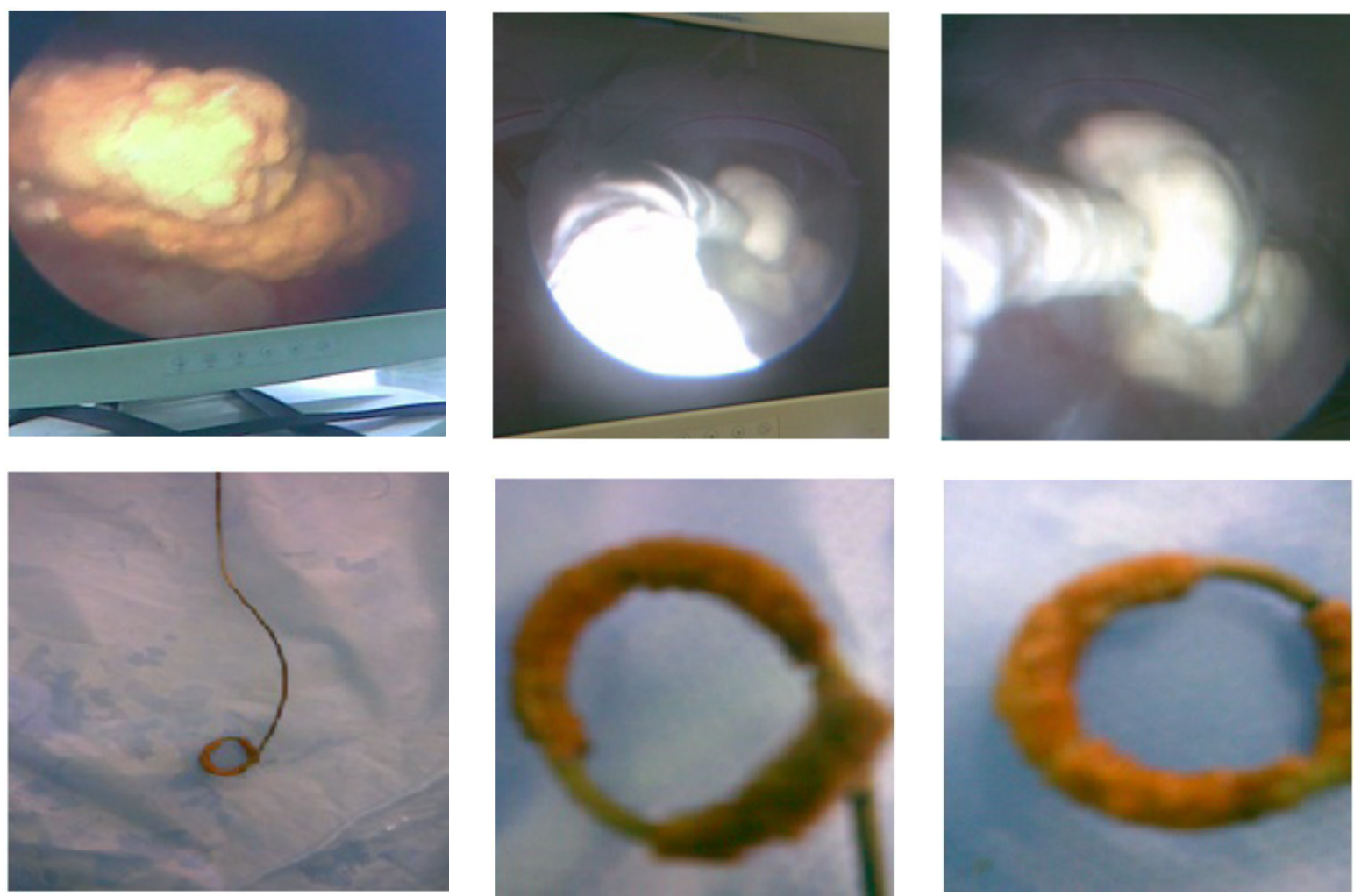

Fig7. Encrusted stents in the remaining two cases (Cystoscopicview) during treatment and removal 
Reporting 5 Cases of Forgotten Ureteral Stent Treated by Endourological Approaches and Citrate Alkaline Therapy

\section{DISCUSSION}

Forgotten ureteral stents were observed in urologic practice due, either, to poor compliance of the patient as to observe medical instructions, or failure of the physician to offer adequate counselling with the patient. These forgotten stents can produce considerable morbidity and mortality, due to extensive encrustation with significant stone burden, knot formation, upward migration and fragmentation $(7,15)$. The deposition of encrusted material on retained ureteral stents can occur in both cases; infected and sterile urine. The mechanism of encrustation in infected urine takes place as result of organic components in the urine crystallizing out onto the surface of biomaterial and becoming incorporated with a bacterial biofilm layer. Urease is produced by the adhered bacteria hydrolyses while the urea produces ammonia. This elevates urinary $\mathrm{pH}$, favouring the precipitation of magnesium, calcium as struvite and hydroxyl apatite $(16,17)$. Although the exact mechanism of encrustation in sterile urine is unclear, it appears to be dependent on the $\mathrm{pH}$, ionic strength and biomaterial hydrophobic properties (18). The degree of encrustation depends on the dwelling time. One study found that $9.2 \%$ of stents became encrusted once placed for less than 6 weeks, while 47.5 of stents that remained in place for 6 to 12 weeks were encrusted, while $76.3 \%$ of stents were encrusted after 12 weeks ( El-Faqih et al,1991) (19). Other factors work also are considered as to increased incidence of encrustations; chronic recurrent stone formers; metabolic predisposition to stone disease, congenital renal anomalies, malignant urinary obstruction and pregnancy (20).Fragmentation is another important complicated factor in terms of the forgotten stents. It is the result of loss of tensile strength, which is due to hardening and degeneration of the stent polymers (21). Retained ureteral stents with encrustation is a challenging problem of endourologists. Very often, multiple endourological approaches are needed because of encrustations and the associated stone burden that may involve bladder, ureter, kidney in addition to, in terms of our cases, alkalinisation therapy of stone formation inhibitors to dissolve and decrease encrustation and stone formation, during treatment. We treated these cases by using ESWL as to the stones and the heavy encrustation of the upper coil of stent and further the alkaline therapy with citrates to decrease the encrustation and stone formation during the treatment of lithotripsy of ureteric stones, bladder stones and encrusted lower coil as well as to alkaline medication with citrate. All respective patients remained free from stones, encrusted stents and their follow ups were uneventful. In the meantime the foregoing treatment remained for six months. Only one female patient remained suffering loin pain, in spite of removal of encrusted stent and her renal isotopes scan showed enlarged non-functional kidney. Nephrectomy was performed with the histopathology reported malakoplakia of the kidney which is a rare inflammatory disease associated with Escherichia coli infection; ( granulomatous interstitial nephritis caused by kidney parenchymal malakoplakia). Our cases were treated via multiple procedures as ESWL, ureteroscopy with lithotripsy, Cystolithotripsy, cystolitholapaxy, and alkaline therapy with potassium and sodium citrate during the whole period of treatment because we believing that citrate is considered as stone and encrustation inhibitors in the urinary tract; refer to literature (22).Selective medical therapy for nephrolithiasis is highly effective in preventing new stone formation. Citrate efficacy for the management of hyperuricosuric calcium nephrolithiasis may stem from inhibitory activity of citrate in respect of calcium and oxalate crystallization. Citrate is an important inhibitor that can reduce calcium stone formation through several mechanisms: citrate reduces urinary saturation of calcium salts via compounding with calcium (23).Citrate directly prevents spontaneous nucleation of calcium oxalate $(24,25)$.Citrate inhibits agglomeration and sedimentation of calcium oxalate crystals $(26,27)$, as well as growth of calcium oxalate and calcium/phosphate crystals. Ultimately, normal urinary citrate levels can enhance the inhibitors effect of Tamm- Horsfall glycoprotein (28). Due to the foregoing, open surgery type is no longer needed in our cases to remove encrusted stents, as we believe that citrates have a role to dissolve part of the encrustation and further decrease stone formation issue and thus encrustation becomes easy to fragment by lithotripsy. 
Reporting 5 Cases of Forgotten Ureteral Stent Treated by Endourological Approaches and Citrate Alkaline Therapy

\section{CONCLUSION}

Encrustation and stone formation in cases of forgotten stents often leads to life threatening complications and pose a challenging management task for the treating surgeon. Alkaline therapy works, in addition to the endourology lithotripsy and ESWL, to decrease the encrustation stent and inhibits new formation of stones in cases of forgotten stent; hence it works to decreasing the need for open surgery.

On light of this situation we deem that it necessary to maintain contact and ensure regular follow-up visits for patients suffering JJ stent placement in order to avoid such dangerous complications in the future. By applying this process, we aim to reduce the patient morbidity and avoid any unnecessary inconvenience thereto.

\section{REFERENCES}

1. Zimskind PD, Fetter TR, WikersonJL.Clinical use of long-term indwelling silicone rubber ureteral splints inserted cystoscopically. J Urol 1967;97:840-4

2. Saltzman B: Ureteral stents. Indications, variations, and complications UrolClin North Am. 1988; 15: 481-91.

3. Chew BH, Knudsen BE,Denstedt JD: The use of stents in contemporary urology.CurrOpin Urol.2004;14;111-5.

4. Hepperlen TW, Mardis HK, KammandelH: The pigtail ureteral stent in the cancer patient. J Urol. 1979; 121: 17-8.

5. Gogas J, Markopoulos C, Kouskos E, Gogas H, Kiriakou V:Metastatic retroperitoneal and mediastinal fibrosis as first sign of recurrence of breast cancer.Eur J Surg.2001;167:715-8.

6. Park DS,ParkJH, Lee YT: Percutaneous nephrostomy versus indwelling ureteral stents in patients with bilateral nongenitourinary malignant extrinsic obstruction .J Endurol.2002;16:153-4

7. Kuno K, MenzinA,Kauder HH, Sison C, Gal D:Prophylactic ureteral catheterization in gynecologic surgery, Urology.1998;52:1004-8.

8. Damiano R, OlivaA,EspositoC, De Sio M, Autorino R, D,Armiento M:Early and late complications of double pigtail ureteral stent. Urol Int.2002;69:136-40.

9. Schulze KA, WettlauferJN,Oldani G: Encrustation and stone formation: complication of indwelling ureteral stents, Urology.1985;25:616-9

10. Mohan-pillai K, Keeley FX Jr,Moussa SA, SmithG, Tolley DA: Endourological management of severely encrusted ureteral stents. J Endourol.1999; 13:377-9

11. FlamTA, Brochard M Zerbib M, Debre B, Steg A: Extracorporeal shock-wave lithotripsy to remove calcified ureteral stents. Urology 1990; 36:164-5.

12. Somers WJ: Management of forgotten or retained indwelling ureteral stents.Urology.1996; 47:431-5

13. Borboroglu PG, Kane CJ: Current management of severely encrusted ureteral stents with a large associated stone burden .J Urol.2000;164:648-50.

14. Lam JS, Gupta M: Tips and tricks for the management of retained ureteral stents .J Endourol.2002;16: 733-41. 
Reporting 5 Cases of Forgotten Ureteral Stent Treated by Endourological Approaches and Citrate Alkaline Therapy

15. Eisner B, Kim H Sacco D: Repeat knot formation in a patient with an indwelling ureteral stent. IntBraz J Urol.2006; 32:308-9

16. Wollin TA, Tieszer C, Riddell JV , Denstedt JD, Reid G:Bacterial biofilm formation, and antibiotic adsorption to ureteral stents indwelling in humans.JEndourol. 1998;12;101-11.

17. Robert M, Boularan AM, El Sandid M, Grasset D :Double-J ureteric stent encrustations: clinical study on crystal formation on polyurethane stents. UrolInt 1997;58:100-4

18. Keane PF , Bonner MC , Johnston SR, Zafar A, Gorman SP : Characterization of biofilm and encrustation on ureteric stents in vivo. Br J Urol.1994;73:687-91.

19. El-Faqih SR, Shamsuddin AB, Chakrabarti A, Atassi R, KKardar AH, Osman MK, et al: Polyurethane internal ureteral stents in treatment of stone patients: morbidity related to indwelling times. J Urol 1991; 146: $1487-91$

20. Lojanapiwat B: Endourological management of severely encrusted ureteral stents. J Med Assoc Thai.2005;88:1203-6.

21. Zisman A, Siegel YI , Siegmann A, Lindner A:Spontaneous ureteral stent fragmentation. J Urol. 1995;153: 718-21.

22. YasinIdweini: Citrate Medication to prevent stent Encrustation in Urolithiasis Therapy: J Urology and Nephrology Open Access 2016;2(3):1-4.

23. Pak CY. Citrate and renal calculi; An update. Miner electrolyte Metab.1994;20:371-377.

24. Primingen GMK, Sakhaee K, Skurle C, Pak CY: prevention of recurrent calcium stone formation with potassium citrate therapy in patients with distal renal tubular acidosis. J Urol 1985; 134:20-23.

25. Sakhee et al: Recent advances in the pathophysiology of nephrolithiasis. Kidney Int. 2009; 75: 585-595.

26. Kok et al: Excessive crystal agglomeration with low citrate excretion in recurrent stones formers. Lancet 1986;1:1056-1058.

27. Tiselius 1993 Fornader, Nilsson. The effects of citrate and urine on calcium oxalate crystal aggregation. Urol. 1993; 21, 363-366.

28. Hess et al: Citrate and calcium effects on Tamm-Horsfall glycoprotein as modifier calcium oxalate crystal aggregation. Am J physiol.1993;265-f784-f791.

Citation: Dr. Yasin Idweini, Ph.D., MD, FEBU “Reporting 5 Cases of Forgotten Ureteral Stent Treated by Endourological Approaches and Citrate Alkaline Therapy". American Research Journal of Urology; 2(1): 1-9.

Copyright (c) 2017 Dr. Yasin Idweini, Ph.D., MD, FEBU .This is an open access article distributed under the Creative Commons Attribution License, which permits unrestricted use, distribution, and reproduction in any medium, provided the original work is properly cited. 